\title{
An investigation on the biotribocorrosion behaviour of CoCrMo alloy grafted with polyelectrolyte brush
}

\author{
Hong-Yu Zhang ${ }^{\text {a }}$, Yu-Jiao Zhu ${ }^{\mathrm{b}}$, Xiang-Yu Hu ${ }^{\mathrm{a}}$, Yan-Fang Sun ${ }^{\mathrm{a}}$, Yu-Long Sun ${ }^{\mathrm{a}}$, Jian-Min \\ $\mathrm{Han}^{\mathrm{c}}$, Yu Yan ${ }^{\mathrm{b},{ }^{*}}$ and Ming Zhou ${ }^{\mathrm{a}}$ \\ ${ }^{a}$ State Key Laboratory of Tribology, Department of Mechanical Engineering, Tsinghua University, \\ Beijing 100084, China \\ ${ }^{b}$ Corrosion and Protection Center, Beijing University of Science and Technology, Beijing 100083, \\ China \\ ${ }^{c}$ Dental Materials Laboratory, National Engineering Laboratory for Digital and Material Technology \\ of Stomatology, Peking University School and Hospital of Stomatology, Beijing 100081, China
}

\begin{abstract}
Surface grafting of polyelectrolyte brush, such as 3-sulfopropyl methacrylate potassium salt (SPMK), on hip implant materials has been reported to reduce the wear of the orthopaedic bearing surface. However, the biotribocorrosion behaviour of the SPMK brush has not been taken into consideration in previous research. In the present study, SPMK was grafted on Co28Cr6Mo alloy through photo-induced polymerization, and the biotribocorrosion behaviour was investigated by a series of frictional-electrochemical tests using a universal materials tester combined with an electrochemical measurement (three-electrode) system. Co28Cr6Mo disk and polyethylene (PE) pin were used as the contact pair, and the lubricants were $0.9 \%$ saline solution $(\mathrm{NaCl})$ and $0.9 \%$ saline solution coupled with $25 \%$ bovine serum albumin (BSA). The results showed that SPMK was successfully grafted on Co28Cr6Mo alloy, which was confirmed by the comparison of Raman spectroscopy and static contact angle of the samples before and after surface modification. The greatly reduced electrochemical parameters such as corrosion current and pitting potential indicated that the corrosion rate of Co28Cr6Mo alloy was significantly reduced following SPMK grafting. Additionally, the frictional-electrochemical coupled measurement performed under reciprocating sliding demonstrated that the lowest corrosion current was obtained for the SPMK-grafted Co28Cr6Mo disk, with $0.9 \% \mathrm{NaCl}$ coupled with $25 \%$ BSA as the electrolyte. It is indicated from the present study that SPMK polyelectrolyte brush can greatly improve the anti-biotribocorrosion properties of Co28Cr6Mo alloy, and thus has potential application on surface modification of hip implant materials.
\end{abstract}

Keywords: Biotribocorrosion, biotribology, surface grafting, hip implant, polyelectrolyte brush

\section{Introduction}

Total hip replacement (THR) is considered as an effective orthopaedic procedure performed worldwide to treat patients with hip disorders, e.g. osteoarthritis, and rheumatoid arthritis. However,

\footnotetext{
${ }^{*}$ Corresponding author: Yu Yan, Corrosion and Protection Center, Beijing University of Science and Technology, Beijing 100083, China. Tel.: +86 010 62332345; Fax: +86 010 62781379; E-mail: yanyu@ustb.edu.cn.
}

0959-2989/14/\$27.50 @ 2014 - IOS Press and the authors. 
following implantation of the hip prosthesis, friction and wear can occur during daily use, resulting in generation of wear debris [1-3]. The wear debris-induced aseptic loosening has been accepted as the main reason for failure of THR [4]. Generally, wear can be produced at both the articulating head-cup interface and also the stem-cement interface, and in literature there are an amount of studies available investigating wear properties of these two interfaces [5-7]. For example, at the stem-cement interface, it is typically fretting wear mechanism caused by a low-amplitude oscillatory movement between polished femoral stem and bone cement [8-10]. Recently, although the use of hard-on-hard bearing systems, such as metal-on-metal and ceramic-on-ceramic configurations [11], has been advocated to greatly reduce the generation of wear debris, the issues with regard to toxic metallic ion release and biotribo-acoustics are considered as major concerns $[12,13]$. Consequently, metal-on-polymer contact, such as CoCrMo alloy-on-polyethylene (PE), is still comprehensively used in clinical situations.

In order to improve the survivorship of THR and the quality of life of the patients, it is essential to reduce the wear generated by the total hip joint system [14,15]. Nowadays, surface grafting of polyelectrolyte brush has been reported as an effective methodology to extend the lifetime of hip prosthesis. In 2010, Kyomoto et al. developed a technique to achieve surface grafting of a biocompatible 2-methacryloyloxyethyl phosphorylcholine (MPC) polymer on CoCrMo alloy [16]. It showed an extremely low friction coefficient at a level lower than 0.01 , which could be attributed to hydration lubrication mechanism [17]. Additionally, it was demonstrated in the study performed by Kobayashi et al. in 2012 that 3-sulfopropyl methacrylate potassium salt (SPMK) showed an even lower friction coefficient than that of MPC when coated on the silicon wafer through surface-initiated atom transfer radical polymerization (ATRP) [18]. Although friction coefficient is documented to be greatly reduced by surface grafting of these polyelectrolyte brushes, it is considered that the hip implant materials experience corrosion under a corrosive environment in the human body $[19,20]$, and the biotribocorrosion behaviour of the polyelectrolyte brushes has not been investigated in previous research. Accordingly, in the present study, an insight into this issue was gained by performing a series of frictional-electrochemical tests using a universal materials tester combined with an electrochemical measurement system.

\section{Materials and methods}

\subsection{Surface grafting of SPMK on CoCrMo alloy}

A total of thirty Co28Cr6Mo disks (ASTM F75 standard specification) were manufactured with a diameter of $10 \mathrm{~mm}$ and a thickness of $3 \mathrm{~mm}$. The Co28Cr6Mo disk was highly polished to achieve a surface roughness value of $10 \mathrm{~nm}$, which was measured by a micro XAM-3D optical interferometer (KLA-Tencor Corp, Milpitas, California, USA) with a scanning area of $0.86 \times 0.64 \mathrm{~mm}^{2}$. This surface was comparable to that of the femoral ball. Then the Co28Cr6Mo disk was cleaned in an ultrasonic bath with acetone for 10 minutes, and immersed in 35\% nitric acid for 30 minutes to dissolute foreign substance residues. Subsequently, the Co28Cr6Mo disk was placed in a tube furnace for irradiation with $\mathrm{O}_{2}$ gas flow at $450^{\circ} \mathrm{C}$ for 60 minutes. 

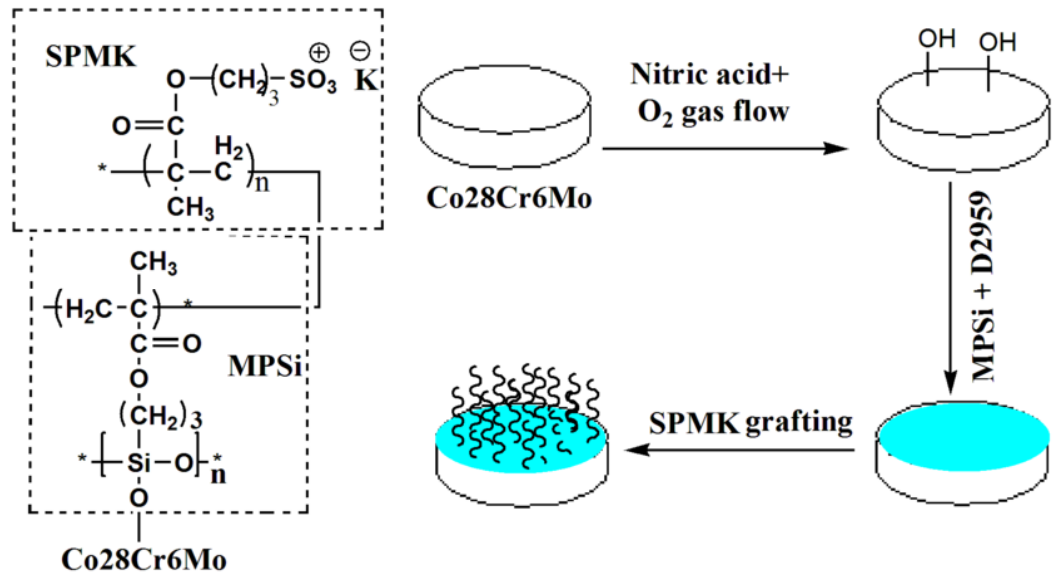

Fig. 1. Schematic illustration for preparation of SPMK-grafted Co28Cr6Mo disk.

The procedure for preparation of SPMK-grafted Co28Cr6Mo disk is shown schematically in Figure 1. Specifically, the pretreated Co28Cr6Mo disk was immersed in an ethanol solution containing 5\% 3methacryloxypropyl trimethoxysilane (MPSi), 1\% succinic acid, and 0.1\% 2-Hydroxy-1-[4-(2hydroxyethoxy)phenyl]-2-methyl-propan-1-one (D2959) overnight for silanization of the trimethoxysilane group. Then the Co28Cr6Mo disk was annealed at $70^{\circ} \mathrm{C}$ in water bath for 3 hours, and transferred to the SPMK aqueous solution at a concentration of $0.5 \mathrm{~mol} / \mathrm{L}$ (prepared by using degassed deionized water). Finally, photo-induced polymerization was initiated through ultraviolet irradiation with an intensity of $5 \mathrm{~mW} / \mathrm{cm}^{2}$ at $80^{\circ} \mathrm{C}$ for 2 hours. After polymerization, the SPMKgrafted Co28Cr6Mo disk was washed with deionized water and ethanol, and dried at room temperature.

\subsection{Surface characterization of SPMK-grafted CoCrMo alloy}

In order to confirm the presence of the SPMK polyelectrolyte brush grafted on the CoCrMo alloy surface, the highly polished Co28Cr6Mo disk and the SPMK-grafted Co28Cr6Mo disk were examined by a LabRAM HR800 Raman spectroscopy (Horiba JY, Ville d'Asq, France) with a wave number range from $400 \mathrm{~cm}^{-1}$ to $4000 \mathrm{~cm}^{-1}$ to detect the functional group vibration of the samples. Additionally, the surface wettability of the highly polished Co28Cr6Mo disk and the SPMK-grafted Co28Cr6Mo disk was evaluated by the sessile drop method employing an OCA-20 contact angle system (Dataphysics Instruments, Filderstadt, Germany). An auto pipette was used to deposit drops of ultrapure distilled water $(2.0 \mu \mathrm{l})$ on the samples, and the static contact angle was measured. A total of ten measurements were performed for each kind of sample, and the average value of the static contact angle was eventually calculated.

\subsection{Biotribocorrosion behaviour of SPMK-grafted CoCrMo alloy}

A custom-made three-electrode cell, as schematically shown in Figure 2, was developed to perform the frictional-electrochemical tests for evaluation of biotribocorrosion behaviour of the SPMK-grafted CoCrMo alloy [21]. The exposed area of the Co28Cr6Mo disk (highly polished and SPMK-grafted), a graphite rod, and a calomel electrode represented the working electrode, the counter electrode, and the 


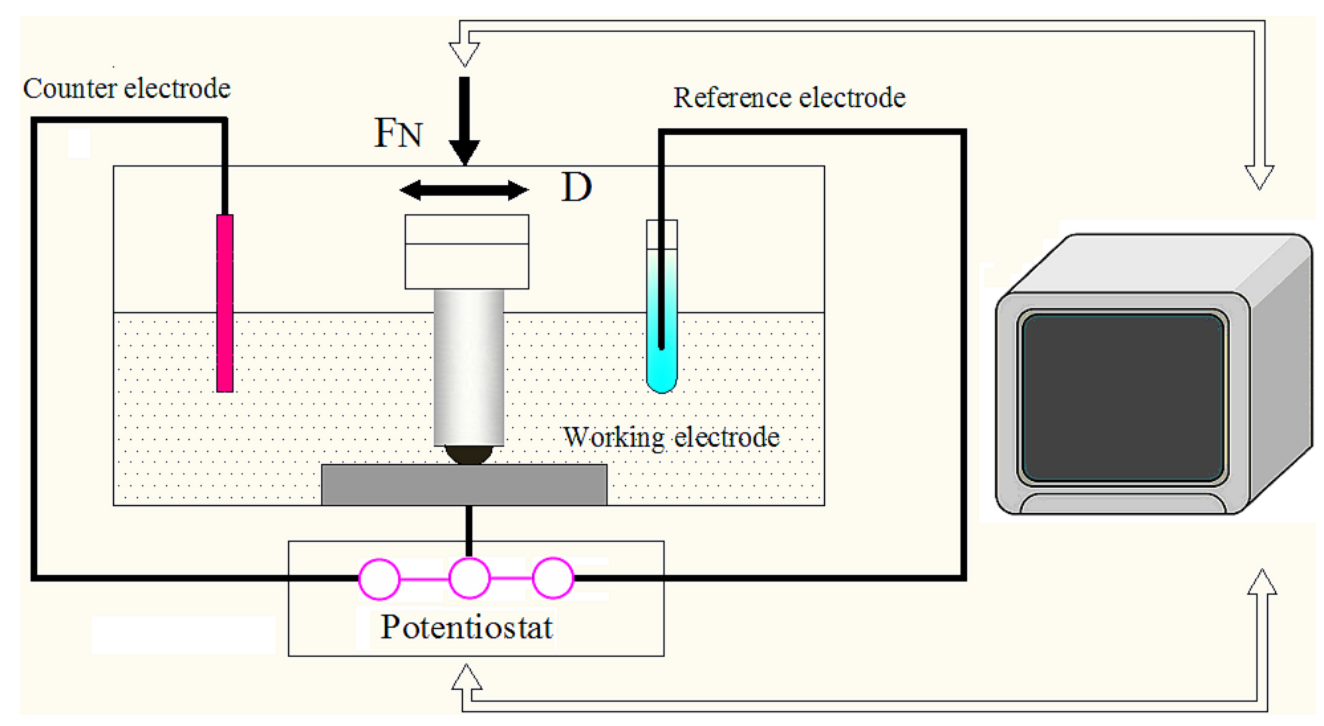

Fig. 2. Development of a three-electrode cell for frictional-electrochemical test.

reference electrode, respectively. The three-electrode cell was further connected with the linear stage of a UMT-II universal materials tester (Centre for Tribology Inc., Campbell, California, USA) for the frictional measurements, and a CHI660e electrochemical measurement system (Chenhua Instruments Inc., Shanghai, China) was used to collect the electrochemical parameters. Two kinds of electrolytes were tested, including $0.9 \%$ saline solution $(\mathrm{NaCl})$ and $0.9 \%$ saline solution coupled with $25 \%$ bovine serum albumin (BSA).

Initially, the static corrosion properties of the Co28Cr6Mo disk (highly polished and SPMK-grafted) was characterized by potentiodynamic polarization curve recorded from $-0.8 \mathrm{~V}$ to $+1.2 \mathrm{~V}$ at a scanning rate of $2 \mathrm{mV} / \mathrm{s}$. Two corrosion parameters, i.e. $I_{\text {corr }}$ (corrosion current) and $E_{b}$ (pitting potential), were obtained from the potentiodynamic polarization curve employing Tafel's method. Subsequently, the biotribocorrosion behaviour of the Co28Cr6Mo disk (highly polished and SPMK-grafted) was investigated through a series of frictional-electrochemical coupled measurements. The frictional test was performed in a reciprocating mode (oscillation amplitude: $4.5 \mathrm{~mm}$ ) at $1 \mathrm{~Hz}$ for a duration of 60 minutes, with Co28Cr6Mo disk and polyethylene sphere pin (diameter: $8 \mathrm{~mm}$; surface roughness: 1.5 $\mu \mathrm{m}$, measured by the micro XAM-3D optical interferometer with a scanning area of $0.86 \times 0.64 \mathrm{~mm}^{2}$ ) as the contact pair, Figure 3. A normal load of $3 \mathrm{~N}$ was applied, which was equivalent to an apparent maximum contact pressure of $37 \mathrm{MPa}$. This was calculated based on the Hertz theory for ball-on-flat configuration [22], Equations (1) and (2). $P$ is the apparent maximum contact pressure, $w$ is the normal load $(3 \mathrm{~N}), R$ is the radius of the pin $(4 \mathrm{~mm}), E_{l}$ and $\mu_{l}$ are the elastic modulus and Poisson's ratio of Co28Cr6Mo (210 GPa and 0.3), and $E_{2}$ and $\mu_{2}$ are the elastic modulus and Poisson's ratio of polyethylene (1 GPa and 0.4$)$.

$$
P=\frac{1}{\pi}\left(\frac{3 w}{2}\right)^{\frac{1}{3}}\left(\frac{E^{\prime}}{R}\right)^{\frac{2}{3}}
$$




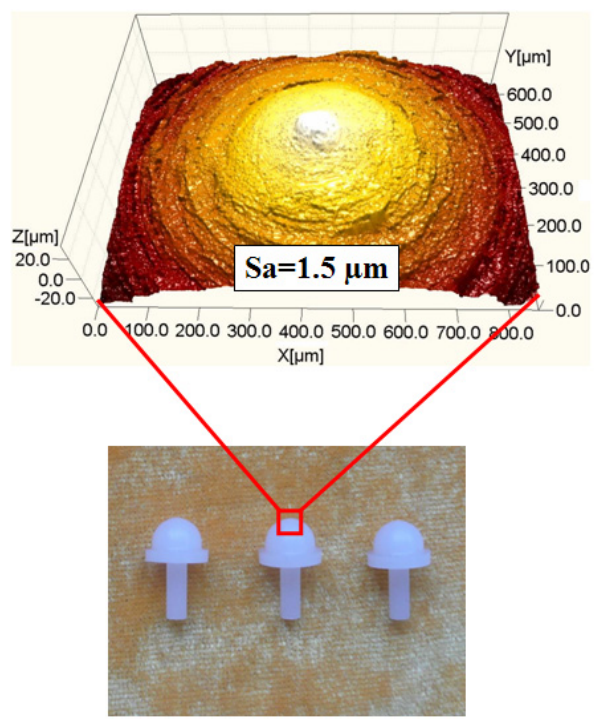

(a)

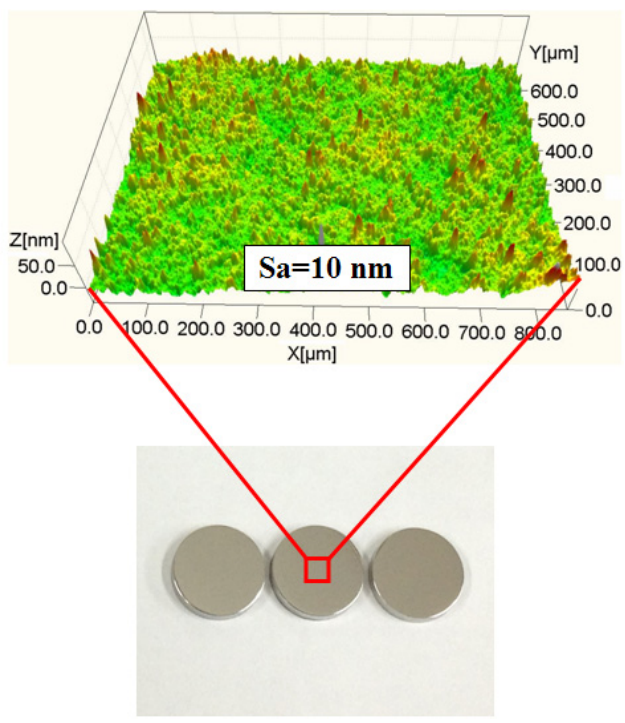

(b)

Fig. 3. Preparation of samples for frictional-electrochemical test: (a) polyethylene pin; (b) Co28Cr6Mo disk.

$$
\frac{1}{E^{\prime}}=\frac{1}{2}\left(\frac{1-\mu_{1}^{2}}{E_{1}}+\frac{1-\mu_{2}^{2}}{E_{2}}\right)
$$

The friction coefficient was recorded during the frictional test, and simultaneously the current-time curve was obtained by the CHI660e electrochemical measurement system under a potential of $+0.2 \mathrm{~V}$ to accelerate corrosion. Note that the open circuit potential (OCP) was monitored for a period of 10 minutes to ensure the electrochemical stabilization of the three-electrode cell. For each measurement, at least three tests were repeated to ensure statistical validity.

\section{Results and discussion}

\subsection{Surface characterization of SPMK-grafted CoCrMo alloy}

The comparison of Raman spectroscopy of the highly polished Co28Cr6Mo disk and the SPMKgrafted Co28Cr6Mo disk was shown in Figure 4. It was obvious that no peaks were detected for the highly polished Co28Cr6Mo disk, whilst the SPMK-grafted Co28Cr6Mo disk was characterized by the presence of several new peaks. The peak at $2950 \mathrm{~cm}^{-1}$ and $1470 \mathrm{~cm}^{-1}$ were attributed to the $\mathrm{C}-\mathrm{H}$ bond, the $1750 \mathrm{~cm}^{-1}$ peak proved the existence of $\mathrm{C}=\mathrm{O}$ bond, and the peaks between $500 \mathrm{~cm}^{-1}$ and $1000 \mathrm{~cm}^{-1}$ demonstrated different kinds of Si-O and O-Si-C bonds. Most importantly, the peak at 1050 $\mathrm{cm}^{-1}$ was a typical absorption of $\mathrm{SO}_{3}^{-1}$, which indicated that SPMK was successfully grafted on the surface of CoCrMo alloy.

The comparison of the static contact angle of the highly polished Co28Cr6Mo disk and the SPMKgrafted Co28Cr6Mo disk was shown in Figure 5. It was clear that the static contact angle of the highly polished Co28Cr6Mo disk $\left(86.9 \pm 0.4^{\circ}\right)$ was significantly larger than that of the SPMK-grafted 


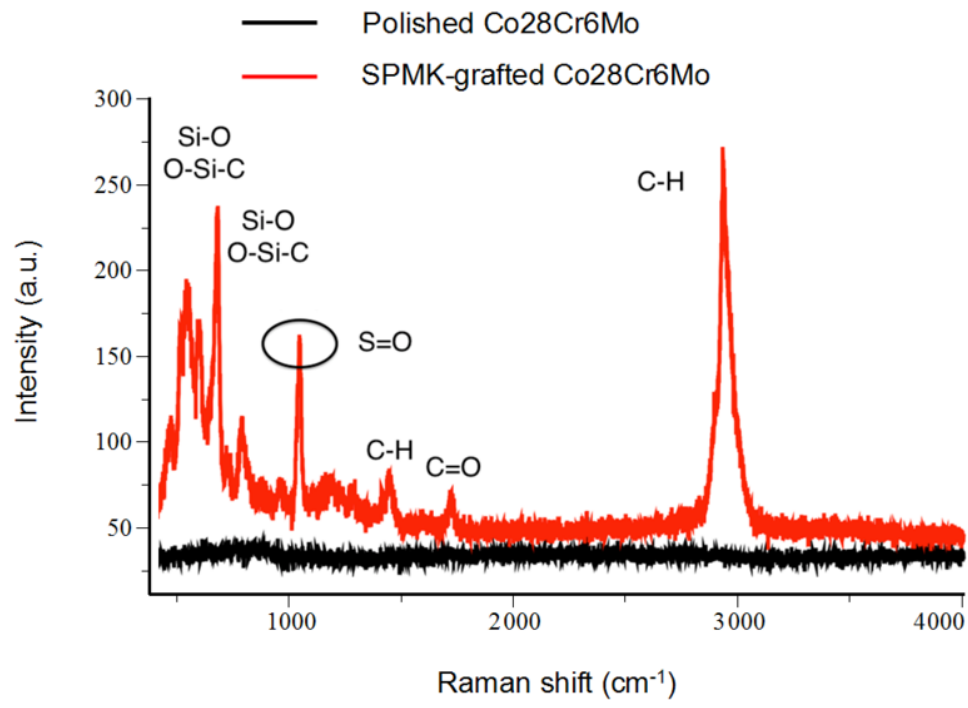

Fig. 4. Raman spectroscopy of highly polished Co28Cr6Mo disk and SPMK-grafted Co28Cr6Mo disk

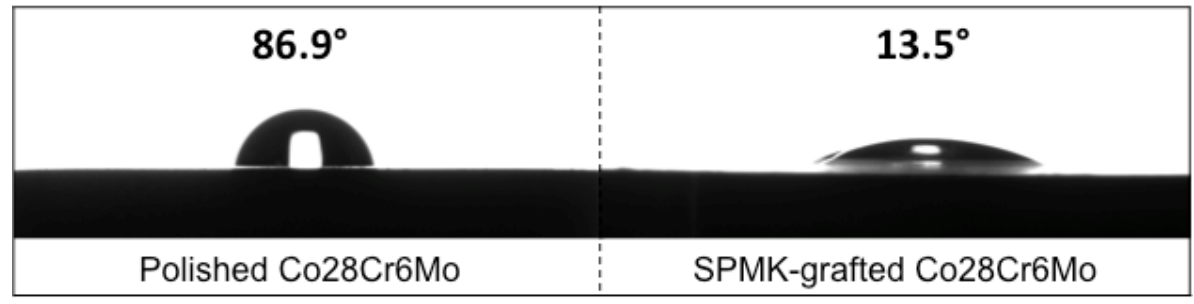

Fig. 5. Static contact angle of highly polished Co28Cr6Mo disk and SPMK-grafted Co28Cr6Mo disk.

Co28Cr6Mo disk $\left(13.5 \pm 1.8^{\circ}\right)$, indicating that SPMK surface grafting on CoCrMo alloy changed the surface wettability of the material from hydrophobic to hydrophilic performance. This result further confirmed the presence of the SPMK polyelectrolyte brush on CoCrMo alloy surface.

\subsection{Biotribocorrosion behaviour of SPMK-grafted CoCrMo alloy}

The potentiodynamic polarization curve of the highly polished Co28Cr6Mo disk and the SPMKgrafted Co28Cr6Mo disk in $0.9 \% \mathrm{NaCl}$ and $0.9 \% \mathrm{NaCl}$ coupled with $25 \% \mathrm{BSA}$ was shown in Figure 6 , and the $\mathrm{I}_{\text {corr }}$ and $\mathrm{E}_{\mathrm{b}}$ values of the samples in these two electrolytes were summarized in Table 1. It was clear from this table that for both $0.9 \% \mathrm{NaCl}$ and $0.9 \% \mathrm{NaCl}$ coupled with $25 \% \mathrm{BSA}$, $\mathrm{I}_{\text {corr-polished CoCrMo }}$

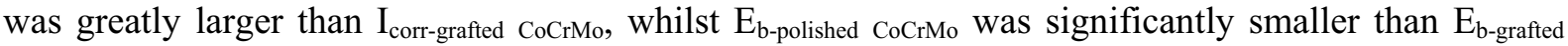
CoCrMo. This result indicated that SPMK surface grafting on CoCrMo alloy could reduce the corrosion rate of the material. Additionally, it was obvious from Figure 6 that the steady state current $\left(\mathrm{I}_{\mathrm{ss}}\right)$ was the lowest for the SPMK-grafted Co28Cr6Mo disk in $0.9 \% \mathrm{NaCl}$ coupled with $25 \% \mathrm{BSA}$, which may be attributed to the formation of a BSA protein film on the surface of the SPMK polyelectrolyte brush, protecting the Co28Cr6Mo disk from corrosion. BSA is a globular protein with a hydrophobic core and hydrophilic surface, and tends to adsorb on all surfaces owing to a gain in conformational entropy [23]. 


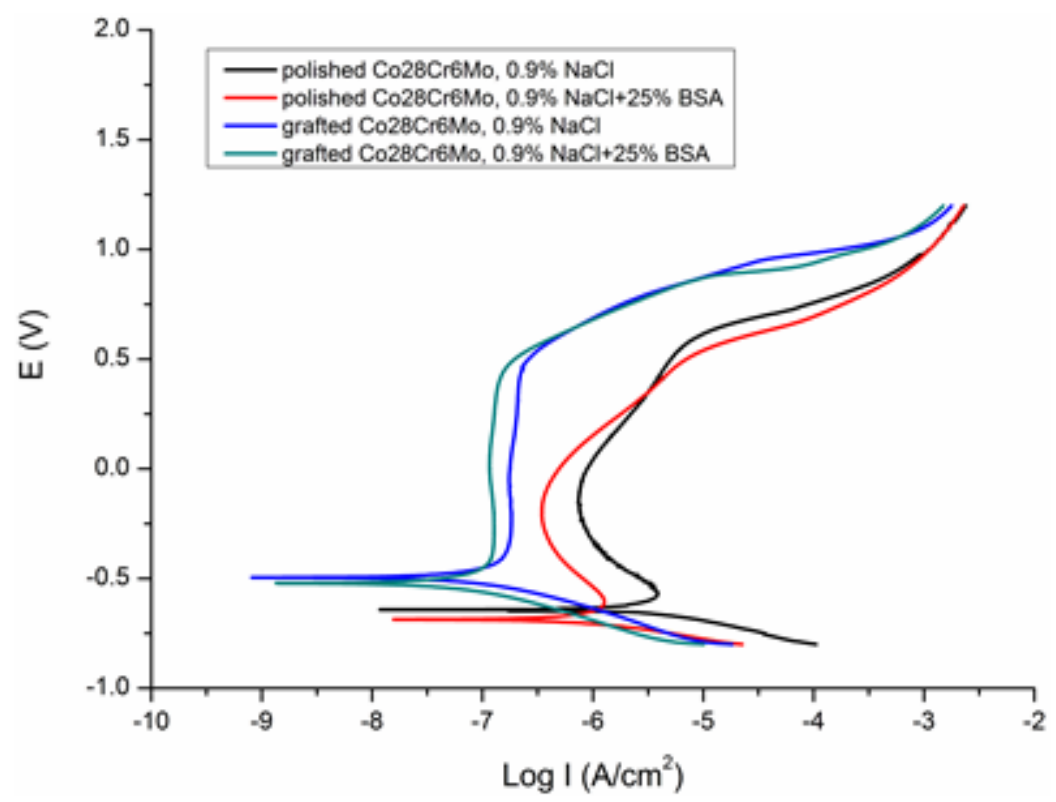

Fig. 6. Potentiodynamic polarization curve of highly polished Co28Cr6Mo disk and SPMK-grafted Co28Cr6Mo disk with $0.9 \% \mathrm{NaCl}$ and $0.9 \% \mathrm{NaCl}+25 \% \mathrm{BSA}$ as the electrolytes.

Table 1

The mean value of corrosion current $\left(\mathrm{I}_{\text {corr }}\right)$ and pitting potential $\left(\mathrm{E}_{\mathrm{b}}\right)$ of the highly polished Co28Cr6Mo disk and SPMKgrafted $\mathrm{Co} 28 \mathrm{Cr} 6 \mathrm{Mo}$ disk with $0.9 \% \mathrm{NaCl}$ and $0.9 \% \mathrm{NaCl}+25 \% \mathrm{BSA}$ as the electrolytes

\begin{tabular}{lllll}
\hline Samples & $0.9 \% \mathrm{NaCl}$ & & $0.9 \% \mathrm{NaCl}+25 \% \mathrm{BSA}$ & \\
& Icorr (10-6 A) & $\mathrm{Eb}(\mathrm{V})$ & Icorr $(10-6 \mathrm{~A})$ & $\mathrm{Eb}(\mathrm{V})$ \\
\hline $\begin{array}{l}\text { highly polished } \\
\text { Co28Cr6Mo disk }\end{array}$ & 4.51 & 0.011 & 1.41 & 0.002 \\
$\begin{array}{l}\text { SPMK-grafted } \\
\text { Co28Cr6Mo disk }\end{array}$ & 0.126 & 0.446 & 0.096 & 0.378 \\
\hline
\end{tabular}

The frictional-electrochemical coupled measurement results, i.e. the current-time curve and the friction coefficient-time curve, were shown in Figure 7. It was clear that the corrosion current for the SPMK-grafted Co28Cr6Mo disk was significantly lower than that of the highly polished Co28Cr6Mo disk in both $0.9 \% \mathrm{NaCl}$ and $0.9 \% \mathrm{NaCl}$ coupled with $25 \%$ BSA. The smallest corrosion current was obtained for the SPMK-grafted Co28Cr6Mo disk, with $0.9 \% \mathrm{NaCl}$ coupled with $25 \% \mathrm{BSA}$ as the electrolyte. This indicated that SPMK surface grafting on CoCrMo alloy could improve the antibiotribocorrosion properties of the material under reciprocating sliding. The friction coefficient-time curve demonstrated that the friction coefficient for the SPMK-grafted Co28Cr6Mo disk was higher than that of the highly polished $\mathrm{Co} 28 \mathrm{Cr} 6 \mathrm{Mo}$ disk in both $0.9 \% \mathrm{NaCl}$ and $0.9 \% \mathrm{NaCl}$ coupled with $25 \%$ BSA. This may be caused due to the increase of surface roughness after SPMK grafting $(0.5 \mu \mathrm{m}$ measured by the micro XAM-3D optical interferometer) on the highly polished Co28Cr6Mo disk. Additionally, it was noted that, with regard to the same contact pair, a higher friction coefficient was obtained when using $0.9 \% \mathrm{NaCl}$ coupled with $25 \%$ BSA as the electrolyte. Previously, it was observed that the friction coefficient was reduced when adding BSA in the lubricant, with either polished titanium alloy or stainless steel and bone cement as the contact pair $[24,25]$. The interaction between 


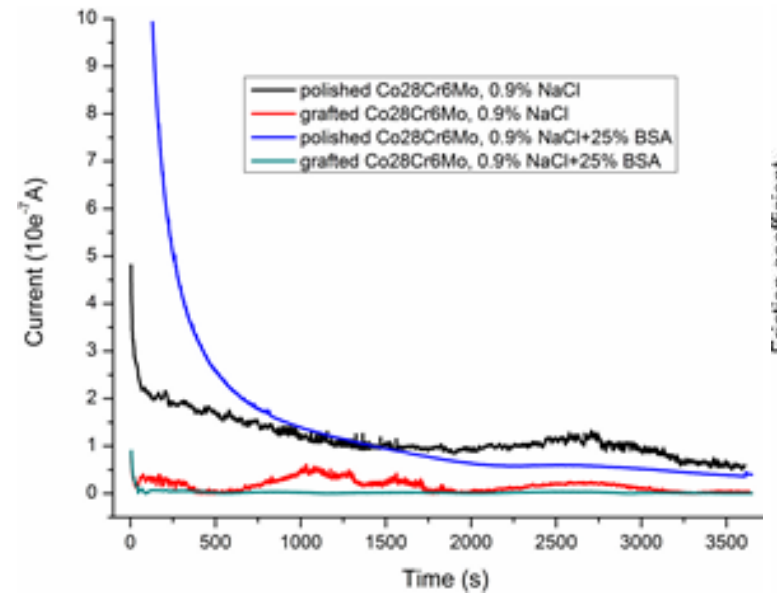

(a)

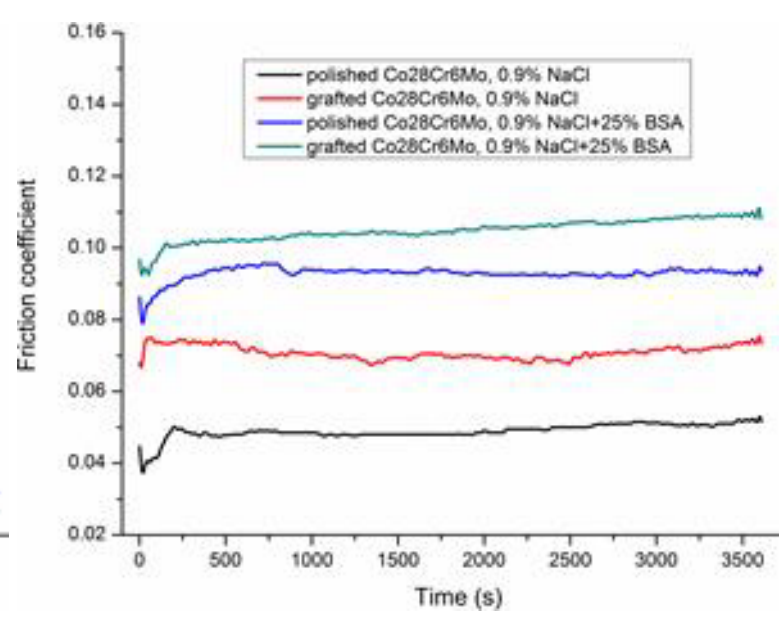

(b)

Fig. 7. Frictional-electrochemical measurements of highly polished Co28Cr6Mo disk and SPMK-grafted Co28Cr6Mo disk with $0.9 \% \mathrm{NaCl}$ and $0.9 \% \mathrm{NaCl}+25 \% \mathrm{BSA}$ as the electrolytes: (a) current-time curve; (b) friction coefficient.

the SPMK polyelectrolyte brush and BSA may contribute to this phenomenon, but further study is required to investigate this issue in more detail.

\section{Conclusion}

In the present study, photo-induced polymerization was developed to graft SPMK polyelectrolyte brush on CoCrMo alloy surface, and the biotribocorrosion behaviour was investigated by a series of frictional-electrochemical tests. The results indicated that both the static and dynamic (reciprocating sliding) anti-corrosion properties of CoCrMo alloy were greatly improved following SPMK surface grafting, therefore representing an effective surface treatment technique for modification of artificial hip implant materials.

\section{Acknowledgement}

This project is supported by National Natural Science Foundation of China (Grant No. 51375008, 51005130), and the Research Fund of State Key Laboratory of Tribology, Tsinghua University, China (Grant No. SKLT12B06).

\section{References}

[1] H. Zhang, L. Blunt, X. Jiang, L. Brown, S. Barrans and Y. Zhao, Femoral stem wear in cemented total hip replacement, Proc. Instn Mech. Engrs, Part H: J. Engineering in Medicine 222 (2008), 583-592.

[2] H. Zhang, L. Brown and L. Blunt, Static shear strength between polished stem and seven commercial acrylic bone cements, J. Mater. Sci. Mater. Medicine 19 (2008), 591-599.

[3] H. Zhang, L. Brown, L. Blunt and S. Barrans, Influence of femoral stem surface finish on the apparent static shear strength at the stem-cement interface, J. Mech. Beh., Biomed. Mater. 1 (2008), 96-104. 
[4] P. Herberts and H. Malchau, Long term registration has improved the quality of hip replacement: A review of the Swedish THR Register comparing 160,000 cases, Acta. Orthop. Scand. 71 (2000), 111-121.

[5] R. Crockett, M. Roba, M. Naka, B. Gasser, D. Delfosse, V. Frauchiger and N.D. Spencer, Friction, lubrication, and polymer transfer between UHMWPE and CoCrMo hip implant materials: A fluorescence microscopy study, J. Biomed. Mater. Res. 89A (2009), 1011-1018.

[6] H. Zhang, L. Brown, L. Blunt, X. Jiang and S. Barrans, The contribution of the micropores in bone cement surface to generation of femoral stem wear in total hip replacement, Tribol. Int. 44 (2011), 1476-1482.

[7] H. Zhang, L. Blunt, X. Jiang, L. Flemings and S. Barrans, The influence of bone cement type on production of fretting wear on the femoral stem surface, Clin. Biomech. 27 (2012), 666-672.

[8] H. Zhang, L. Brown, S. Barrans, L. Blunt and X. Jiang, Investigation of relative micromotion at the stem-cement interface in total hip replacement, Proc. Instn Mech. Engrs, Part H: J. Engineering in Medicine 223 (2009), 955-964.

[9] H. Zhang, L. Brown, L. Blunt, X. Jiang and S. Barrans, Understanding initiation and propagation of fretting wear on the femoral stem in total hip replacement, Wear. 266 (2009), 566-569.

[10] H. Zhang and M. Zhou, The influence of protein concentration on the biotribological properties of the stem-cement interface, Bio-Med. Mater. Eng. 24 (2014), 173-179.

[11] A. Hatton, J.E. Nevelos, A.A. Nevelos, R.E. Banks, J. Fisher and E. Ingham, Alumina-alumina artificial hip joints. Part I: A histological analysis and characterization of wear debris by laser capture micro-dissection of tissues retrieved at revision, Biomaterials 23 (2002), 3429-3440.

[12] Z.K. Hua, Y.W. Fan and Z.M. Jin, A biotribo-acoustic testing method for ceramic orthopaedic biomaterials, Tribol. Int. 71 (2014), 1-6.

[13] Z.K. Hua, H.H. Zhang, Y.W. Fan and Z.M. Jin, Development of a BiotriboPOD testing methodology for the wear evaluation of orthopaedic biomaterials, Royal Society of Chemistry Adv. 4 (2014), 19987.

[14] H. Zhang, L. Blunt, X. Jiang, L. Brown and S. Barrans, The significance of the micropores at the stem-cement interface in total hip replacement, J. Biomat. Sci.-Polym. E. 22 (2011), 845-856.

[15] Z.M. Jin and D. Dowson, Bio-friction, Friction 1 (2013), 100-113.

[16] M. Kyomoto, T. Moro, K. Saiga, F. Miyaji, H. Kawaguchi, Y. Takatori, K. Nakamura and K. Ishihara, Lubricity and stability of poly(2-methacryloyloxyethyl phosphorylcholine) polymer layer on Co-Cr-Mo surface for hemi-arthroplasty to prevent degeneration of articular cartilage, Biomaterials 31 (2010), 658-668.

[17] J. Klein, Hydration lubrication, Friction 1 (2013), 1-23.

[18] M. Kobayashi, M. Terada and A. Takahara, Polyelectrolyte brushes: A novel stable lubrication system in aqueous conditions, Faraday Discuss 156 (2012), 403-412.

[19] Y. Yan, A. Neville and D. Dowson, Biotribocorrosion-an appraisal of the time dependence of wear and corrosion interactions: I. The role of corrosion, J. Phys. D: Appl. Phys. 39 (2006), 3200-3205.

[20] Y. Yan, A. Neville and D. Dowson, Biotribocorrosion-an appraisal of the time dependence of wear and corrosion interactions: II. Surface analysis, J. Phys. D: Appl. Phys. 39 (2006), 3206-3212.

[21] M.T. Mathew, T. Uth, N.J. Hallab, R. Pourzal, A. Fischer and M.A. Wimmer, Construction of a tribocorrosion test apparatus for the hip joint: Validation, test methodology and analysis, Wear. 271 (2011), 2651-2659.

[22] S.Z. Wen and P. Huang, Principles of Tribology, John Wiley \& Sons Inc., New York, 2011.

[23] M. Roba, M. Naka, E. Gautier, N.D. Spencer and R. Crockett, The adsorption and lubrication behaviour of synovial fluid proteins and glycoproteins on the bearing-surface materials of hip replacements, Biomaterials 30 (2009), 20722078.

[24] H.Y. Zhang, J.B. Luo, M. Zhou, Y. Zhang and Y.L. Huang, Biotribological properties at the stem-cement interface lubricated with different media, J. Mech. Beh. Biomed. Mater. 20 (2013), 209-216.

[25] H.Y. Zhang, S.H. Zhang, J.B. Luo, Y.H. Liu, S.H. Qian, F.H. Liang and Y.L. Huang, Investigation of protein adsorption mechanism and biotribological properties at simulated stem-cement interface, J. Tribology, Transactions of American Society of Mechanical Engineers 135 (2013), 032301, 1-10. 